\section{Discovering the right treatments for diabetes (2), blood's pressure, and relating diseases}

\author{
Alaa Hussein Al-Darraji* \\ Assistant Professor, Department of Chemistry, College of Science, Iraq
}

\begin{abstract}
For last decades diabetes type 2, blood's pressure (especially hypertension) and relating diseases are most serious problems for much people over the world. These diseases are not like other different diseases then for studying them very accuracy, this research choose sample from different societies a small city called "Al-Mejar Al-Kabeer" in south of Iraq. People of this city do same activities than other Iraqi cities and during the last twenty years the only change happened in this city is; they changed them drinking water from tap water to commercial water called it R.O. Population of this city is about 70000 persons and about 20000 from them have diabetes that means more than $28 \%$ from all people of this city have this disease, this ratio or may be more for hypertension.
\end{abstract}

Results of this research show that the main reason for diabetes type 2, blood's pressure and relating diseases is civilization. It shows according good evidences that the right treatment for diabetes, hypertension and relating diseases is about $5 \mathrm{~g} /$ day potassium ions $\left(\mathrm{K}^{+}\right)$with less amounts from sodium ions such as 2:1. In fact, insulin forming from known amino acids so each body need sufficient amounts from these acids therefore the right treatment for diabetes type 2 is not only potassium it must there sufficient amounts from proteins each day or each three days.

References of this research indicated; Diabetes and hypertension are well understood but this research find that these diseases need a chemist touch to be fully understood.

\section{More Information}

*Address for Correspondence: Alaa Hussein Al-Darraji, Assistant Professor, Department of Chemistry, College of Science, Iraq, Tel: 009647802564429; Email: Alaa_h_k@yahoo.com; Alaa.hussein@uomisan.edu.iq

Submitted: 01 August 2019
Approved: 14 August 2019
Published: 15 August 2019

How to cite this article: Al-Darraji AH.

Discovering the right treatments for diabetes (2), blood's pressure, and relating diseases. Arch Pharm Pharma Sci. 2019; 3: 026-041.

DOI: dx.doi.org/10.29328/journal.apps. 1001015

Copyright: (c) 2019 Al-Darraji AH. This is an open access article distributed under the Creative Commons Attribution License, which permits unrestricted use, distribution, and reproduction in any medium, provided the original work is properly cited

Keywords: Diabetes; Hypertension and potassium

Check for updates

\section{Introduction}

Diabetes is chronic polyuria resulting from various metabolic disorders. In most cases, the polyuria results from a high concentration of glucose in the renal tubule [1]. Diabetes mellitus result primarily from inadequate secretion of insulin or the inability of tissues to respond to insulin. Insulin-dependent diabetes mellitus (IDDM), also called type (1) diabetes mellitus, affects approximately $3 \%$ of people with diabetes mellitus and results from diminished insulin secretion. Noninsulin-dependent diabetes mellitus (NIDDM), also called type (2) diabetes mellitus, results from the inability of tissues to respond to insulin. NIDDM usually develops in people older than 40-45 years of age, although the age of onset varies considerably. A strong genetic component exists in the disease, but its actual cause is known. NIDDM is more common than IDDM. Approximately $97 \%$ of people who have diabetes mellitus have NIDDM [2].

However, Blood pressure is a measure of the force blood exerts against blood vessel walls [2]. Two pressures are recorded: (1) Systolic pressure is the peak arterial BP attained during ventricular systole. (2) Diastolic pressure is the minimum arterial BP between heartbeats. For a healthy person, arterial BP is written as a ratio of systolic over diastolic pressure: $120 / 75$ [1].

Above diseases are widely spread in the world, according to global news a high ratios from them in the south of Iraq. In fact, Iraq ministry of health declared that $40 \%$ of Iraqi people are suffering from high Blood pressure while $10 \%$ of them are suffering from diabetes, and these ratios are for people with ages between 25-65 years old.

Diabetes type two is a major serious problem for millions over the world therefore in this research the right explanation for this unlikely disease. This introduces by three scientific experimental ways; Firstly, a small city with about 70 for the last 20 years nothing change in them lives except they change them drinking water and this modifying water does not have potassium ions for this diabetes type 2 ratio increased from about $2-3 \%$ before 2003 to $28.5 \%$ after 
2003, secondly, a chemically particularly method show the relation between glucose, insulin and cell's membrane in normal cases and in diabetes cases for understanding this disease and hypertension in more accuracy, thirdly, there are two unusual studies American and Japans studies show the relation between potassium and diabetes type 2 but they did not explain them results that low serum potassium cause diabetes while this research introduce the right explanation about the reason of diabetes type 2 and its right treatment. This research do this in three ways for ending this unlikely disease. In addition, it has the right treatment for hypertension and why it is happen moreover another explanation.

\section{Experimental part}

For the last fifteen years people of "Al-Mejar Al-Kabeer" city do same activities like other cities in Iraq with no any differences so it is a good place for study different diseases that have high score such as diabetes mellitus or hypertension. This city have one big hospital and according to its records the population about 70000 persons and about 20000 from them have diabetes mellitus type 2 , this for hypertension too.

It is a fact that people of this city sharing each other in only two factors; Water and Air. Most people over the world sharing each other by these factors only.

In addition, percentage of Potassium ion $\left(\mathrm{K}^{+} \%\right)$ was calculated in different water samples (six samples) by using flammable atomic absorption technique in Chemistry Department - College of Science. Six samples were collected from different sources of water in Missan city. Four of these samples are from different filters (including Reverse Osmotic (R.O.) waters and deionized waters), which means they produce from different filtration techniques. The rest two samples were from tap water that produced from normal purification processes.

\section{Results}

People of "Al-Mejar Al-Kabeer" city are about 70000 persons and about 20000 from them have diabetes mellitus, rates of diabetes $=28.57 \%$, the fact of this truth, it is a high score.

Percentages yield of six samples of this research are; for four samples (filtered water including R.O. samples and deionized waters) are $=$ Zero, there are no Potassium ions in the four different water samples while the tap water contain about $3.1-3.2 \mathrm{mg} / \mathrm{L}$ of this ions $\left(\mathrm{K}^{+}\right)$.

\section{Discussion}

There must be a scientific reason explaining the high rate of diabetes mellitus in "Al-Mejar Al-Kabeer" city, there are so many people got diabetes about $28.57 \%$ after 2003 while before this year it is not like this! It is small city and its people sharing each other by two factors only; Water and
Air. Air mainly has different gases $\left(\mathrm{O}_{2}, \mathrm{~N}_{2}\right.$ and $\left.\mathrm{CO}_{2}\right)$. There is no evidence indicate that these gases have any relation with diabetes mellitus or hypertension.

Then other factor should cause diabetes mellitus. Due to known reason Iraqi people in south of Iraq after 2003 used commercial water called "R.O" reverse osmosis which is deionized water instead of them tap water. Results of this research show that this waters do not have necessary ions such as Potassium ions. There are good evidences show that there is a relation between potassium-sodium ions with diabetes mellitus and hypertension.

Science reach this relation between sodium and glucose in penetration process into different cells $[1-4,18]$, that Glucose and $\mathrm{Na}^{+}$bind to different sites on a $\mathrm{Na}^{+}$- glucose symporter located at the apical surface. $\mathrm{Na}^{+}$moves into the cell down its electrochemical gradient and "drag" glucose with it. Therefore, the greater the $\mathrm{Na}^{+}$gradient the more glucose enters; and if $\mathrm{Na}^{+}$in extracellular fluid is low, glucose transport stops [14]. For this, $\mathrm{Na}^{+}$is more important for glucose transport into different species cells.

Then after each meal billions from glucose molecule should be at extracellular fluid at the blood of the body, they need same number from sodium ions. The question is; from where the body get this amount from sodium ions! Moreover, if these sodium ions enter all body cells then them concentration must be higher in intracellular than at extracellular. This is incorrect and real status indicated it is an opposite from real status of the body [2], concentration of sodium ions is higher at extracellular than intracellular while potassium ions has an opposite status.

It so clear that sodium-potassium ions have major rule in glucose transport into different cells. $\mathrm{K}^{+}-\mathrm{Na}^{+}$ATP-ase is well known transporter when three $\mathrm{Na}^{+}$enter into different cells two $\mathrm{K}^{+}$are get out $[1-4,18]$, this relation is known and it is responsible for entering all glucose molecules into different cells of the body.

In fact, People of Al-Mejar Al-Kabeer have normal lives before or after 2003 until now, specialists people of its hospital indicate that before 2003 people that have diabetes or hypertension were not more than $2 \%$ while nowadays it is about $28.57 \%$ for diabetes and about same for hypertension. This differences is abnormal $2 \%$ to $28.57 \%$ in only fifteen years 2003-2018, there must be a scientific reason caused this!

The only difference that happened in Al-Mejar Al-Kabeer is that they replaced them drinking normal tap water by R.O. water (deionized water), before 2003 people of this city drink normal tap water. Scientific reason of R.O. waters they do not have potassium ions, this research results show that these waters do not contain these ions. Therefore, this the only difference happened in Al-Mejar Al-Kabeer for the last twenty years. 
A conclusion of above results that potassium ions play major rule in glucose penetration process into different cells of human body that means they have major rule in diabetes and hypertension diseases.

Diabetes rates for Al-Mejar Al-Kabeer is $28.57 \%$ including all ages 1-65 years of all people types and this rates for other cities in the south of Iraq or may be more than this. In addition, countries of Arab gulf and other countries show same rates or may be more. Actually this rates for all the world as WHO indicated, they are scary rates and they are because most people over the world drink commercial water does not contain or it contains less amounts from important ions "potassium ions".

It is so clear that people of Al-Mejar Al-Kabeer about 70000 do not have any effecting factor since 2000 the only change happened in them lives during the last fifteen years 2003-2018 is that they replaced drinking tap water by deionized water (R.O. water). This kind of water does not contain potassium ions as mentioned before then it is a fact that less level of potassium ions in the body cause diabetes and hypertension. From 2000 to 2018 rates of diabetes disease are increased from about $2-3 \%$ to 28.57 because of decreasing of potassium ions.

Above fact comes from 15 years with about 70000 samples about 20000 from them have diabetes and about same for hypertension, after these results it is more than enough for insuring from the right treatment for diabetes and hypertension which is about $5 \mathrm{~g} /$ day potassium ions.

However, chemistry cannot accept this because there is no clear explanation for the binding of glucose with sodium ion! Chemistry have many different bonds such as covelant, ionic, hydrogen...etc. which one glucose bind to sodium when they entering different cells through cell's membrane?! And also why glucose bind to sodium and does not with potassium? Sodium prefer ionic bonds does glucose-Na bond is ionic? There are another questions, chemistry should answer them for more insuring from the right treatment for diabetes type 2 and hypertension diseases. Explaining these questions give additional evidences adding to above one for being more insuring from diabetes and hypertension treatment.

Results of this research indicate that the tap water contain potassium ions about $(3.1-3.2 \mathrm{mg} / \mathrm{L})$ while filtered water samples (including R.O. samples, deionized waters) that produced from different filters processes, are not contain potassium ions. Potassium ion is most important for human body [1-4]. There is a good relation between diabetes and relating diseases with potassium ions [1-18].

As it is mentioned before, above evidences and explanations are more than enough about the right treatment for diabetes type 2 and for hypertension but there is addition evidence should be added as follow:
For healthy person, there is a certain concentration of glucose in its blood which is about $100 \mathrm{mg} / 100 \mathrm{ml}$ [2]. According to references [1-6], insulin assist glucose molecules for passing through specific channels in plasma membranes binding with sodium, then it will be converted to glucose-6-phosphate inside human's cells. Understanding this process should lead to understand exactly what happen for people to have diabetes and relating diseases. Following scheme should clarify this explanation Scheme 1:

Scheme 1 is a chemical explanation for entering blood glucose with sodium inside body's cells by assisting of insulin [3]. Scheme 1 clarify how glucose enter through cell membrane by insulin with sodium and what it gives inside body cells. It is obvious that chemical structure of entering glucose molecules do not change during entering process and insulin is responsible for making glucose enter body's cells. By another words, glucose molecules stay as they are when entering body cells by assisting of insulin hormone. Therefore, the questions are; How insulin make glucose molecules able to enter cell membrane for Glycolysis process? And why glucose does not entering cell's membrane without assisting of insulin? Finally what changes kinds should occur in glucose molecules to be able to enter human's cells? These questions will lead to understand the reason of diabetes, hypertension and relating diseases.

Diabetes mellitus is well known since about three thousand years, its name came from Greek word siphon which mean secretion of amounts of urine, while mellitus means sweet taste, this mean that diabetes mellitus in Greek language indicate secretion of urine with sugar.

However, glucose represent the main energy source for human body, the fact of different bodies, they are mainly depend on carbohydrate metabolism in them daily actions more than other metabolisms such as fatty acids or amino acids. For this, metabolism of carbohydrate is well studied by many scientists and researchers during the last two centuries. Then there are many facts about carbohydrate metabolism and other relating process this research depend on them and they are as follow:

1. In the normal person, there are mainly certain levels of glucose and insulin in its blood [1-4].

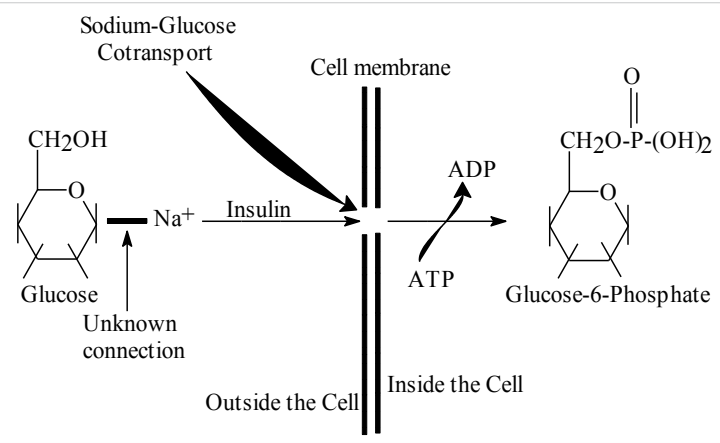

Scheme 1: Illustrate how blood glucose enter body cells and results of this entrance. 
2. After each meal, glucose level in the blood is increased and as a response of this insulin will increase too, Pancreas secrete high amounts of insulin as a response of existing of high amounts of glucose in human blood [1-4].

3. As a life evidence, human will lose most of its energy after 5-7 hours without food.

4. Both molecules glucose and sodium are important for entering glucose inside human's cells through cell membrane [1-4].

5. Potassium Chloride is important treatment for diabetes (Diabetic Ketoacidosis) [8].

6. Different compounds of cells membranes are well studied [1-6].

7. Glucose structure and its properties are well studied [1-4].

8. Insulin function and its chemical structure are well studied [1-4].

9. Glucose molecule become glucose-6-phosphate after entering human cells [1-4].

10. Insulin is the hormone that making glucose entering through protein channel to be inside the cell as indicated before.

11. Inside different cells, Glucose converted to be a $\mathrm{CO}_{2}$, $\mathrm{H}_{2} \mathrm{O}$ and ATP molecules which are the source of energy for human body.

12. Univalent positive ions such as $\mathrm{Na}^{+}$and $\mathrm{K}^{+}$are absorbed by human intestine relatively faster than other bivalent ions, molecules or compounds [1-6].

13. For each two potassium ions entering human cells, there are three sodium ions are getting out from these cells [1-6].

14. References indicated that there is a relation between diabetes and low level of serum potassium [7,15-17].

Therefore, Glucose paths inside human cells are well studied by a lot of scientists for so many years ago. This because that glucose is the main molecule of Bio-energy for human body by carbohydrate metabolism as it is mentioned before. However, It is a fact that above (14) points contain the solution of diabetes disease in addition to what mentioned before but this solution needs more than above points, it is needs chemist's touch.

Scheme 1 showed that glucose convert to glucose-6phosphate after passing through cell membrane by insulin hormone. According to chemistry science, glucose when converted to glucose-6-phosphate it reacts with ATP molecule inside human cells. This reaction is a simple and it will explain later.

From outside (blood) to inside human's cells glucose entering by helping of insulin molecules. Therefore, there are two important aspects for entering process of glucose inside different human cells, these are; cells membranes and insulin. In fact, these aspects can be explained according to references [1-4] and chemistry science as follow:

\section{Cell's membrane}

According to references [1-4], it is called plasma membrane, it is a lipid bilayer composed of phospholipids and cholesterol with proteins that extend across or are embedded in either surface of the lipid bilayer. However, its function it controls entry and exit of substances; receptor molecule function in intercellular communication; marker molecules enable cells to recognize one another [1,2]. In fact, depending on chemical rules the blood is a polar liquid cannot be interacted with lipid bilayer membrane.

There are two different compounds are across through plasma membrane; certain specific non-lipid-soluble molecules (polar molecules) such as glucose or different ions (sodium) diffuse through membrane channels, while lipidsoluble molecules (non-polar molecules) diffuse directly through plasma membrane [2]. Therefore, according to this, glucose with sodium entering human cells through process called Facilitated diffusion that is a carrier-mediated or channel-mediated moves substances into or out of cells from a higher to a lower concentration. This process does not require metabolic energy to transport substances across the plasma membrane [2].

Potassium ions are entering inside the cell through channel protein for three sodium ions exit out, and also these exiting ions are entering the cell again with glucose molecules through carrier-protein. In another words, potassium ions provide extracellular by sodium ions to assist glucose to enter the cell.

Reference [2] indicate that Plasma membrane consists from $45 \%$ - $50 \%$ lipids, $45 \%$ - $50 \%$ proteins, and $4 \%-8 \%$ carbohydrates. As it is mentioned before, non-polar molecule enter body cells through them membrane while polar molecules enter body cells through membrane channels (channel proteins and carrier proteins) [1-4].

Depending on cells positions in the body, there are many channels in their membrane [1-4]. Also same references indicate that there are two types of membrane channels; carrier proteins and channel proteins. The first one (carrier protein), do not need energy and the rate at which molecules or ions are transported directly proportional to their concentration gradient up to the point of saturation, when all carrier proteins or channel are occupied. Then the rate of transport remains constant at its maximum rate. In addition, 
the transport rate is limited by the number of carrier proteins and the rate at which each carrier protein can transport solutes, when the number of molecules outside the cell is so large that the carrier proteins are all occupied, the system is saturated and the transport rate cannot increase [2].

The second type of membrane channels is channel proteins which are one or more integral protein arranged so that they form a tiny channel through the plasma membrane. References suggested that hydrophobic regions of the proteins face outward toward the hydrophobic part of plasma membrane, and the hydrophilic regions of protein face inward and line the channel [1-3]. In fact, references suggested that this indication is for all channels in plasma membrane.

By another words, membrane's channels are located in membrane proteins regions of plasma membrane, they consisting of membrane proteins called Integral proteins consist of regions made up of amino acids with hydrophobic (R) groups and other regions of amino acids with hydrophilic (R) groups. The hydrophobic regions are located within the hydrophobic part of the membrane, and the hydrophilic regions are located at the inner or outer surface of the membrane or line channels through the membrane [2].

This means that above two channels (carrier proteins and channel proteins) are arranged in plasma membrane, the hydrophobic regions of them are located within hydrophobic part of the membrane, and the hydrophilic regions are located at the inner or outer surface of the membrane or line channels through the membrane.

Depending on above points cell's membrane or as it is called plasma membrane does not have any relation with diabetes or hypertension. Different ions and glucose can enter this membrane so easily without any complications and without spending any energy kinds. Therefore, cell's membrane does not have any role in glucose penetration through it then insulin have the major role as follow:

\section{Insulin}

This molecule is well studied by a lot of scientists during the last years, it is protein hormone synthesized as inactive precursor, and active molecule is derived by proteolysis. For instance, insulin an important metabolic regulator is generated by proteolytic excision of a specific peptide from proinsulin [4]. Insulin is consist from known 51 amino acids while proinsulin is consist from known 86 amino acids [4]. Chemical structure of human insulin showed that it has 51 different amino acids, also they divided to three groups; hydrophilic, hydrophobic and in between amino acids [4]. This can be illustrated as in the following table:

A scientific fact, that when dropping few drops of fatty acid or phospholipid in a beaker of $250 \mathrm{ml}$ water with stirring, these drops will collect together forming micelle or bigger drops (emulsion). This depend on the concentration of fatty acid or concentration of phospholipids compounds. However, fatty acids, phospholipids compounds...etc. are collecting together because of them solubility in water and water molecules force them to be together forming micelles or emulsions. Water molecules force lipid molecule to be one big drop.

However, human's Blood is an aquatic solvent and insulin has hydrophobic moieties as showed in table 1, therefore, above fact should be happened for insulin molecules in human blood because two reasons; firstly, human blood is moving solvent or stirring solvent. Second, human insulin have two different groups of amino acids; hydrophilic and hydrophobic amino acids. Then the blood should force insulin molecules to form micelles, the hydrophilic amino acids will be outer micelles while hydrophobic amino acids will be inner. In addition, this may happen because insulin is a small molecule [2], therefore it should form micelles does not emulsions in the stirring blood.

Therefore, (23) amino acids of insulin will be inward insulin's micelles while (25) amino acids will be outward insulin's micelles in the blood. According to table 1, there are additional three amino acids "in between amino acids", they will be either outward or inward insulin's micelles and they do not effect above (25) hydrophilic amino acid that they are outward insulin's micelles.

It should be noticed that cysteine will form three disulfide bridges in insulin structure [1-4]. These bridges give insulin its structure and stability also these bridges and forces of blood (aquatic solution) will force insulin to be a micelle which is like a tiny drop in the blood, once again (25) amino acids are outward this drop while (23) will be inward this drop. The fact of the rest (3) amino acids (in between) will be outward insulin's micelle because them (R) groups are ($\mathrm{NH}_{3}{ }^{+}$and $-\mathrm{OH}$ ), hydrophilic groups but they are few amino acids (3) comparing with (25) so they do not effect insulin function.

Therefore, total outward amino acids is 25 amino acids, there are (6 Cys) in disulfide bridges, resulting (19) free (R) groups of insulin outward amino acids.

\begin{tabular}{|c|c|c|c|c|c|}
\hline \multicolumn{5}{|c|}{ Table 1: Showed names and numbers of human insulin (51) amino acids. } \\
\hline Hydrophilic amino acids & Hydrophobic amino acids & In between amino acids \\
\hline Name & Number & Name & Number & Name & Number \\
\hline Cysteine & 6 & Leucine & 6 & Tyrosine & 2 \\
\hline Glutamic acid & 4 & Valine & 4 & Lysine & 1 \\
\hline Glutamine & 3 & Phenylalanine & 3 & & \\
\hline Threonine & 3 & Glycine & 4 & & \\
\hline Serine & 3 & Isoleucine & 2 & & \\
\hline Asparagine & 3 & Tryptophan & 2 & & \\
\hline Histidine & 2 & Alanine & 1 & & \\
\hline Arginine & 1 & Proline & 1 & & \\
\hline Total amino acids $=25$ a.a. & Total amino acids = 23 a.a. & Total amino acids = 3 a.a. \\
\hline
\end{tabular}


These amino acids by dismissing them number in insulin are; Glu., Gln., Thr., Ser., Asn., His, and Arg. therefore, insulin molecules in blood should be as drops approximately such as following figure by neglecting numbers of amino acid in these drops (micelles of insulin):

For complete above figure there is about 13 carbon atoms attached to withdrawing atoms like oxygen or nitrogen atoms, these carbons should have partial positive charges. This means in insulin there are 13 positive centers or there are 13 electrophile centers easy to attach by any nucleophile such as oxygen, nitrogen,...etc. In addition, Insulin molecule is amphipathic molecule because it consisting from hydrophilic and hydrophobic amino acids as illustrated before therefore molecule like insulin should form micelle in moving aquatic solvent (the blood).

According to figure 1 there are two different groups of hydrophilic amino acids in insulin structure; amino acids with carbonyl group attached to hydroxyl group $(-\mathrm{OH})$ or attached to amine group $\left(-\mathrm{NH}_{2}\right)$ in addition to Arginine. The second group are; Thr. and Ser. This means that in hydrophilic group of insulin micelle, there are (25) amino acids minus (6) amino acids of (Cys.) because of disulfide bridges, the rest amino acids of hydrophilic amino acids are (19 amino acids). These (19) amino acids will be (13) amino acid of above first group of carbonyl group with other molecules plus Arg., while the second group as illustrated above are (6) amino acids of Thr. and Ser.

Depending on figure 1, the second group of above three amino acids are two of them (Serine and Threonine) are from alcohols types compounds and glucose is from this type too, there is not interactions between same compounds (glucose with these compounds).

Therefore, according to chemical structure and properties of insulin's amino acids, insulin in human blood will be as drop showing (13) amino acids that containing carbonyl groups with amine or hydroxyl moieties or carbon attached to nitrogen by double bonds as carbonyl. In addition, according to chemical structure of glucose there is a one way for Glucose to react with insulin or according to drops of insulin micelle in the blood, they have one reacting kind to react with glucose.

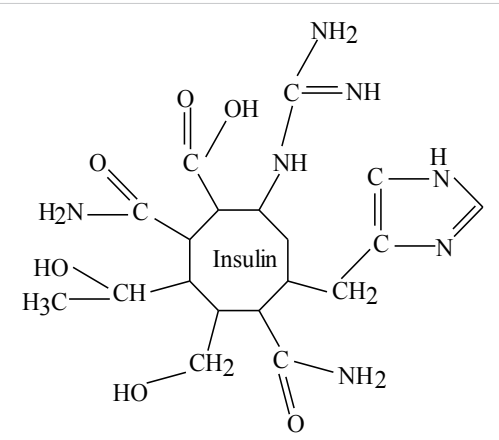

Figure 1: Estimation micelles structure of insulin molecule in the blood.
After knowing that the cell membrane (Plasma membrane) should have another mechanism for entering glucose molecules and knowing insulin chemical structure in the blood and it shows different (13) amino acids outward insulin micelles. Then it should be understand how glucose enter human cells.

\section{How glucose enter human cells}

For normal person, glucose enter its different cells by three steps as follow:

Reaction of glucose with insulin: In this step, it will show how glucose react with insulin. This happened because both concentrations of above compounds are increased in human's blood after each meal or after another conditions such as after drinking sugars syrups or after eating fast foods (i.e. different sandwiches) ...etc.

However, according to chemistry science, glucose has six carbon atoms, the first one is aldehyde moiety but there are some facts indicate that aldehyde moiety in glucose is inert does not react with other compounds such as other aldehydes therefore scientists [4] suggested that glucose has circular structure as showed before in scheme 1 . Glucose have five hydroxyl group and the last one (carbon number six) is primary alcohol free group to attach another molecules. In chemistry language, glucose have two different groups either hydroxyl groups $(-\mathrm{OH})$ or carbonyl group (-(CO)-). Hydroxyl groups are attacking while carbonyl group is accept attacked by another moieties.

In addition to above facts which lead to scientific suggestion of circular structure of glucose molecules in human blood [4]. When glucose reacts with ATP after passing through cell membrane it reacts through carbon number 6 the hydroxyl moiety even that ATP have many oxygen atoms able to react with glucose through carbon number. There are another fact show that carbon six of glucose is more active than other carbons specially carbon 1 such as in glycoside or acetyl formation reaction without deactivation of carbon six by acidic catalyst this reaction does not happen, as mentioned before phosphor ester formation for glucose and fructose inside the cell or outside, this reactions is so important for human bio-energy. This clear facts show that the attached moieties of glucose are hydroxyl $(-\mathrm{OH})$ of carbon number six.

However, glucose has active primary alcohol $(-\mathrm{OH})$ while insulin has different groups accepted attack of hydroxyl group, they are as mentioned before carbonyl groups with amine and hydroxyl moieties in addition to Arg. In fact, there is only one reacting type can be happened between glucose and insulin's different groups which are 13 electrophiles attached by 13 nucleophiles of glucose. This reaction is same of esterification reaction that is depend on the structure of each reactant (glucose and insulin). Structure of glucose which showed before is showed carbon number six in glucose as the following figure 2 . 


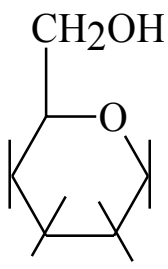

Figure 2: Glucose chemical structure.

Figure 2 showed that oxygen of carbon number (6) primary alcohol is more active than other oxygen atoms of glucose structure. This because that each carbon atom of glucose molecule from 1 to 4 have hydroxyl group attached to each one of them, these hydroxyl group make them less activity than hydroxyl number six because oxygen is more electronegativity than carbon. Four oxygen atoms of carbon 1-4 of glucose are withdrawing molecules leading to electron density for them is less than hydroxyl number six which is free and connected to carbon without oxygen atom carbon number (5). In addition to what it mentioned before there is another factor which is that oxygen group of hydroxyl moiety of carbon six is outer the plane of glucose than other hydroxyls in stereochemistry of glucose molecule (Haworth projection formula).

Therefore, oxygen of hydroxyl moiety of carbon six of glucose molecule has good electron density and it is more active than other oxygen atoms in glucose molecule for reacting with insulin as follow:

As illustrated before there are (13) amino acids for five types of amino acids which are; Glu., Gln., Asn., His and Arg., are hydrophilic amino acids in outer face of insulin. Above amino acids structure contain different groups so they can be presented as following figure 3 except His. which has another structure:

Where,

$$
\mathrm{R}=-(\mathrm{R}-\mathrm{OC}),(\mathrm{R}-\mathrm{NH})-\mathrm{CH}-\left(\mathrm{CH}_{2}\right)_{\mathrm{n}}-; \mathrm{n}=1,2 \text { or } 4
$$

$\mathrm{X}_{1}=\mathrm{O}$ or $\mathrm{NH}$.

$\mathrm{X}_{2}=\mathrm{O}^{-}$or $\mathrm{NH}_{2}$.

Then the reactions steps or mechanisms of total reactions will be:

Step 1: A 13 molecules from Glucose should attach 13 positive centers in insulin molecule or a 13 good nucleophiles (glucose molecules) should attach 13 electrophile centers of insulin moiety as following figure 4.

Step 2: Positive charge on oxygen mean unstable molecule so proton transfer should be happened as figure 5 .

After attaching of glucose figure 3 and below one are just intermediate steps because they are highly unstable molecules give the products very fast.
Step 3: This step represent rearrangement step for above intermediate molecules as follow figure 6.

A summary of above steps; after each meal or after another statuses a glucose concentration in the blood be at maximum lead the body to excrete insulin from $\beta$-cells. Glucose is good nucleophile attach insulin which has 13 electrophiles centers and this interaction is well studied as it is mentioned before. In addition, this interaction produce highly unstable molecules resulting the products as faster as it is possible. Interaction product of step (1) that oxygen atom of glucose molecule should have positive charge unstable atom so proton transfer should happen for making this oxygen be more stable otherwise impossible for oxygen to bind with three atoms. However, the reason that all products are intermediate because there is another unstable status still there at the product of glucose attached. This status that $\left(-\mathrm{X}_{1} \mathrm{HNa}^{+}\right)$moiety $=10\left(-\mathrm{OHNa}^{+}\right)$and $3\left(-\mathrm{NH}_{2} \mathrm{Na}^{+}\right)$, the numbers 10 and 3 refers to number of amino acids in insulin $\mathrm{Glu}=4, \mathrm{Gln}=3, \mathrm{Asn}=3$, while His and $\mathrm{Arg}=3$. Both $\left(-\mathrm{OHNa}^{+}\right.$ or $-\mathrm{NH}_{2} \mathrm{Na}^{+}$) moieties are highly unstable and they should have positive charges as $\left(-\mathrm{O}^{+} \mathrm{HNa}^{+}\right.$or $\left.-\mathrm{N}^{+} \mathrm{H}_{2} \mathrm{Na}^{+}\right)$because both atoms $\mathrm{O}$ and $\mathrm{N}$ bind to more than its ability, then they reduce this by forming double bond as shown in figure 5 . Last step is rearrangement for all intermediates resulting

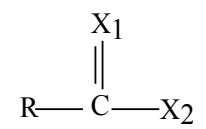

Figure 3: Chemical structure of each moiety of 12 amino acids in insulin molecule.

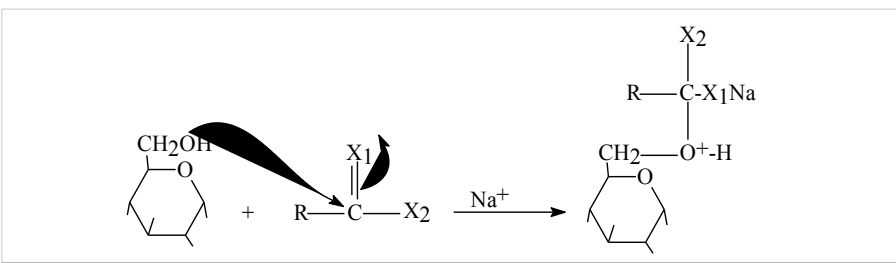

Figure 4: Step one glucose molecules attach insulin center.

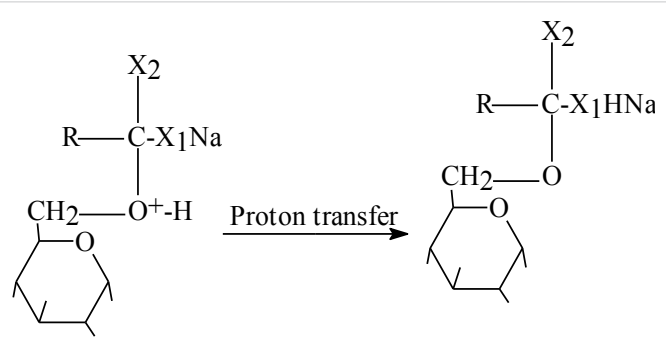

Figure 5: Step two proton transfer in intermediate status.

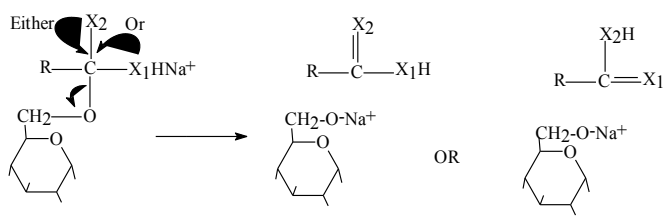

Figure 6: Step three rearrangement of intermediate molecules. 
one products which is sodium gluconate. Noticing that all 13 amino acids have carbon center has partial positive charge make them good electrophiles, 10 from them have same $\mathrm{R}$ groups carbonyl moieties binding to oxygen in carboxylate groups in Glu $=4$ while the rest amino acids $=6$ are carbonyl moieties binding to amines moieties $\left(-\mathrm{NH}_{2}\right)$. His. and $\mathrm{Arg}$ have different $\mathrm{R}$ groups they have carbon centers but they binding with two amine groups and these centers are more electrophile than above 10 amino acids because nitrogen more electronegativity than oxygen.

In both products of last step figure 6 is sodium gluconate which is the right result. Therefore when glucose attach insulin all steps happen together to produce insulin again and sodium gluconate means resulting known products glucose bind to sodium through ionic bond.

Different references of this research [1-5] and others show that glucose bind to sodium ions for entering different cells but they do not explain this binding? This was mentioned before and this research discover the kind of this bind which is "ionic bond".

There are a another additional evidences should be clarified as follow:

Reaction of Glucose with insulin is look like esterification reaction which means is reversible reaction so when it happens in aquatic liquid (the blood), the product GlucoseInsulin complex do not produce because of water molecules which attached its ester bond to produce reactants again (sodium gluconate and insulin). This means there are no products of esterification reaction for glucose-insulin interaction in aquatic liquid, this property is known for all esters compounds in aquatic liquid.

Both molecules; Glucose and insulin are relatively big molecules so this factor increase hydrolysis of Glucose-Insulin molecule producing reactants again without producing another products.

Following equation can be written for glucose-insulin reaction:

Glucose-insulin intermediates $\longrightarrow$ Glucose-6-O- $\mathrm{Na}^{+}+$ Insulin- $(\mathrm{H})_{11} \ldots$.

Then, total reaction of glucose with insulin should be:

Insulin +13 Glucose $13 \mathrm{Na}+13 \mathrm{Na}+13$ Sodium-Gluconate + Insulin- $(\mathrm{H})_{13} \ldots$

Equation (2) is the main reaction that happened between glucose with Insulin by catalyzing of sodium ion. Insulin react and produce without changing in its structure so it is like other catalysts and also it act as reactant, actually it is so important as reactant for above reaction equation (2). It is so obvious that the main action of Insulin molecules is putting sodium atoms at carbon 6 of Glucose molecules.
This indicates that human get glucose from outside its body while it gets second reactant "insulin" from inside. Both reactants differ in them sources but they are so important for producing Bio-energy for human and other like creatures to do them different actions.

According to above equation (2), above explanations and figures, sodium ion is so important for entering glucose inside human different cells. It has two important physiological rules in glucose-insulin reaction, one of them it is necessary for activating insulin molecules for reacting with glucose. This indicates that decreasing of sodium ions in human blood should decrease insulin activity. While second rule, is make sodium-Gluconate salt more stable for making forward reaction of reaction (2) is more likely than backward reaction.

As a result, Sodium is so important for reaction (2) so before going to next step (step two) of how glucose enter human cells, importance of sodium should be explained in glucose-insulin reaction. According to chemistry science, glucose have active hydroxyl group connected to carbon number six, this group have high electron density should attack atoms have either totally positive charge or partially positive charge. Insulin chemical structure contain carbon atoms have partial positive charges that happened due to electronegativity differences between carbon (less electronegativity) and $\left(\mathrm{X}_{1}\right)$ (high electronegativity) in $\left(\mathrm{R}-\left(\mathrm{C}^{\delta+}=\mathrm{X}_{1}\right)-\mathrm{X}_{2}\right)$ as it is mentioned before.

Then glucose-insulin reaction must happen but what should happen when the main reaction happen without sodium! Glucose should react with insulin even in absence of sodium ions because both molecules are at high blood's concentration level, both molecules have good nucleophiles (glucose) and good electrophiles (insulin) then them reaction must happen but it should be slow and its products are differ than main reaction with sodium equation (2), this should be as in the following scheme (2) (mechanism):

Scheme 2 intermediate is highly unstable because both atoms of $\mathrm{X}_{1}=\mathrm{O}$ or $\mathrm{NH}$, are unstable must return to produce insulin again removing glucose molecules due to them molecule size. In addition, $\mathrm{X}_{1}$ cannot get any other positive atoms rather than $\mathrm{Na}^{+}$such as $\mathrm{H}^{+}$because it does not exist in the blood.

Equation of scheme 2 should be:

$$
\text { Insulin + Glucose } \longrightarrow \text { Insulin + glucose }
$$

In absence of sodium ions the main reaction should happen electrophile of glucose carbon six should attach 13 nucleophiles centers of insulin resulting unstable intermediates producing the reactants again. This means without sodium, insulin and glucose stay as they are in the blood. It is important to mention that without sodium or hydrogen ion $\left(\mathrm{H}^{+}\right)$the main reaction does not occur. 


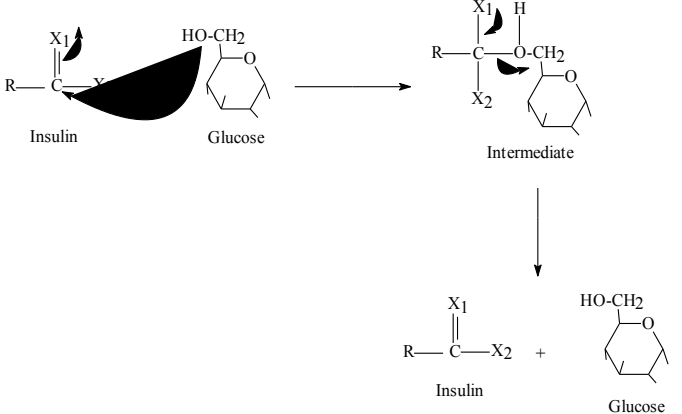

Scheme 2: glucose-insulin reaction without sodium

This because that sodium have relatively small volume can participate in the main reaction while potassium have relatively big volume difficult to participate in the main reaction. Moreover, blood's $\mathrm{pH}$ is 7.2-7.4 this means there is no hydrogen ions $\left(\mathrm{H}^{+}\right)$.

Without sodium ions insulin do not react with glucose and it has a little half life time so it should hydrolyzed in the blood releasing different amino acids that undergo different paths in human's bodies or other like bodies. One of these paths is by hydrolyzed to produce ammonia which converted to Urea by the liver resulting high urea concentration in diabetes patients as these patients show.

Without sodium above products should produce from glucose-insulin reaction. These products are totally differ than products of equation (2) (with sodium ion). However, above equation without sodium ions is slow rather than original reaction (2). In fact, each product of above reactions indicates important issue should be discussed each one alone as in the follow:

First product is, "glucose" which indicate that glucose enter the reaction and produced as it is without any change. Glucose stay as same as it is in the blood so it cannot be able to enter human cells. In another words, insulin's action is to prepare glucose for entering human cells, but without sodium insulin does not do its action, (13) molecules of glucose enter above reaction and produced from it as same as they were which means this will lead to increase glucose levels in blood caused diabetes disease. This means without sodium diabetes disease should occur.

The second product in hydrolysis of insulin are keto acids from amino acids that lose them amine moieties increasing urea molecules in the blood. Kidneys should be tired and less activity in diabetes patients than normal people. This situation keep happen in diabetes patients until it became more serious such as in case of "Diabetic Ketoacidosis" when blood $\mathrm{pH}$ is become acidic because of hydrogen ions of insulin. Treatment of this situation is Bicarbonate as sodium bicarbonate $[8,9]$. This means they gave the patients the right treatment "sodium ions" and this came from medical experimental tests.
Another product is ammonia molecules $\left(\mathrm{NH}_{3}\right)$ from insulin they secrete from insulin molecule to the blood. This happens in case of absence of sodium ion as illustrated before. Each insulin molecule should produce ammonia molecules so for 10000 molecules of insulin, there are a lot of ammonia molecules are produced. These producing molecules will convert by Urea cycle to be urea molecules by urea cycle as following equation: (6).

$2 \mathrm{NH}_{3}+\mathrm{CO}_{2}+3$ ATP $\longrightarrow \mathrm{H}_{2} \mathrm{~N}-\mathrm{CO}-\mathrm{NH}_{2}+\mathrm{H}_{2} \mathrm{O}+3 \mathrm{ADP} \ldots$

Urea

References indicated that in diabetic patients there are high levels of Urea molecules in its blood compare with normal people [8,10-12]. A high Urea levels in blood is just indication for diabetic disease [10-12] and it is a good evidence for explaining of above evidences.

Therefore, above three points and above explanation indicate that; by absence of sodium ions in glucose-insulin reaction; glucose levels in the blood should increase, blood $\mathrm{pH}$ will decrease to acidic levels and ammonia (Urea) levels will increase. In another words, in absence of sodium ions from glucose-insulin reaction diabetes disease should happen.

However, before going to second step of how glucose enter human cells there are three important notices should be clarified as in the follow:

Diabetes disease is not mean that all glucose-insulin reactions that occur in human's blood do not have sodium ions! It means that sodium ions are not enough for all reactions of glucose-insulin that happened in human's blood. This indicates that for diabetes's patient there are two reactions types happen in its body, and these are reactions (2 and 3). These reactions should happen in different percentages in diabetic patient depending on amount of sodium ions in the blood. Therefore, there are different degrees for diabetes in diabetic patients. This because that diabetic patient can talk, walk, think, breath,...etc. doing different actions which means it have high percentage of its body cells get sodiumGluconate while very low cell's percentage of its body do not get sodium-Gluconate molecules. Because if all body's cells do not get sodium Gluconate this lead to death directly in few minutes due to that in this case acidity of the blood should increase may be less than 5 while human die at $\mathrm{pH}=6.8$ ! Glucose's concentration will be more than $500 \mathrm{mg} / \mathrm{dL}$ or much more in the first minutes leading to many disorders in human body resulting death. In addition, concentration of urea molecules should increase much more than what body can stand with leading to death too. All these factors should kill any person with just few minutes or may be less.

However, this if all human's body cells do not get sodiumGluconate. Therefore, in diabetic patients there are two reactions happen in its body but their percentages depends 
on sodium concentrations in its blood. If this concentration is high and enough for following reaction then there is no diabetes and following reaction should occur:

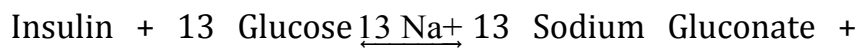
Insulin- $(\mathrm{H})_{13} \ldots \ldots$.

Or if concentration of sodium ions are not enough then above reaction should happen by high ratio and below reaction should happen in low ratio:

Glucose + Insulin $\longleftrightarrow$ Glucose + Insulin

In fact there are another good evidences relating to above facts "medical rules" should be clarified in later sections because they represent an ugly crime happened and still happening in different hospitals over the world.

The second notice is that human body built from about $10^{14}$ different cells and half or more of these cells are not placed near blood circulations. Therefore, how all these cells get necessary compounds such as sodium-Gluconate! This fact leads to important question about how all body's cells get same amounts from sodium Gluconate or other important atoms or molecules! Some of these cells should be faster in getting of sodium Gluconate than others and also third group do not get sodium Gluconate at all because they do not placed near blood. In fact, products of glucose-insulin reaction with sodium ions (equation 2) are not distribute equally for all cells of human body.

The fact of human's body is in equilibrium with its surroundings factors and its cells are in equilibrium with each other. These two equilibrium statuses make human live on this earth and any change in these statuses means death for human beings. Surroundings factor are so strong against different changes while equilibrium of human's cells is not strong enough against different changes. Body's cells equilibrium means that when cells placed near blood's circulation they get necessary ions and compounds such as sodium Gluconate, then concentrations of these ions and compounds should increase inside these cells while groups or series of neighboring cells are not. Equilibrium means that body's cells near blood get necessary ions and compounds then they give these ions and compounds to neighboring cells which will give them to next cells and...etc. until all body's cells get same amounts from important ions and same amount form different compounds. Therefore, body's cells equilibrium means that necessary ions and compounds are moving from one cell to another until all cells get same amounts from them. In fact, even water molecules and other tiny molecules should move to all body cells by this process. There is another equilibrium status which is between different contents of body's cells with contents of the blood from different ions and compounds.

Differences between cells in them containing ions and moieties atoms bind to atoms have high electronagativity. Therefore, hydrogen bonds should occur between glucose molecules and water molecules in the blood as it is showed in the following figure 7 .

Figure 7 show hydrogen bonds that occurred between glucose with water molecules. These bonds are not the only bonds that glucose should do, because blood contain different molecules such as proteins, lipids, enzymes, hormones ...etc. and they have different moieties such as $\mathrm{O}, \mathrm{N}, \mathrm{H}$ and $\mathrm{S}$ atoms. Therefore, because of glucose is highly soluble in blood, it should bond with different blood's molecules not just with water by hydrogen bonding or other interactions.

Above hydrogen bonds of glucose and different blood's molecules prevent glucose from entering human's different cells through their membranes. Or by another words, human's blood catch glucose molecules as they are by hydrogen bonds and prevent them to enter different cells. This reason why glucose does not enter body cells without assistance of insulin. This because that insulin does not change structure of glucose only, it also broke all hydrogen bonds of glucose make it free to enter body's cells. This means that insulin has two jobs; it convert glucose to be a salt "sodium Gluconate" and it broke hydrogen bonds that happen between glucose and different blood's molecules (water molecules and others blood's molecules).

There is additional factor addition to hydrogen bonding which is solubility of glucose is more than sodium Gluconate because sugar are more soluble in water than salt. This means that glucose as sugar prefer to stay in blood rather than leave it while glucose as sodium Gluconate (salt) prefers to leave blood rather than stay in it.

According to above figures and schemes, Insulin reacts with glucose by few steps resulting convert glucose to be sodium Gluconate and it helps glucose to be free for bonding with sodium to form sodium Gluconate which is able to leave human's blood for entering human cells through cells membranes.

Second step is how cell's membranes let sodium Gluconate pass through! As it is mentioned before, Cell's membrane consist mainly from lipids (triglycerides and cholesterol) and proteins [1-3]. Proteins of cell's membrane are forming from hydrophobic and hydrophilic amino acids. The fact is that hydrophobic part of membrane's proteins has relatively same chemical properties of lipids part of the same membrane. This because that both of them are formed from same atoms carbon and hydrogen.

Another fact, cell's membrane is located between two hydrophilic solutions; outside of it the blood and inside it Cytoplasm. Additional fact is that lipids of cell membrane are phosphoglycerides and cholesterol, these molecules have hydrophilic moieties such as phosphate groups (have oxygen 
negative ions) and cholesterol molecules have hydroxyl groups. Therefore, hydrophobic part of cell's membrane are lipids and hydrophobic amino acids of proteins, while hydrophilic part is hydrophilic amino acids of protein and hydrophilic moieties of lipids molecules (phosphoglycerides and cholesterol) of cell's membrane.

Different molecules of Cell's membrane are contacting with blood and cytoplasm which are aquatic liquids so as it is mentioned before. References [1-4], indicated that hydrophilic moieties of all cell's membrane are located outward the membrane contacting with blood and cytoplasm while inward cell's membrane are hydrophobic moieties.

Addition fact is that; all atoms of cell's membrane are moving especially they are placed between two aquatic moving liquids one of them is blood continues moving liquid. Internal spaces between atoms of cell's membrane are continually changing depending on how they connect each other. For all molecules of cell's membrane, hydrophobic moieties have more internal spaces than hydrophilic one. This because that there are many interactions such as hydrogen bonding between hydrophilic different moieties lead to decrease internal spaces while in case of hydrophobic moieties repulsion will increase internal spaces. For this Sodium Gluconate as salt can pass through cell's membrane so easily because it a salt and it passes through three areas; hydrophilic two areas (outward and inward the membrane) and hydrophobic which is inward the membrane. References [1-3], indicates that the basic structure of plasma membrane are determined by its lipids.

There are important points should be noticed for above indication:

Chemical information indicate that penetration activity of two molecules are; $\mathrm{NaCl}>\mathrm{C}_{6} \mathrm{H}_{12} \mathrm{O}_{6}$. According to chemical properties of these molecules, they differ in polarity, $\mathrm{NaCl}$ is more polarity than glucose because it is a salt with full negative and positive charges while glucose does not have. Glucose have five high electronagativity atoms (oxygen atoms) so it have high electron density on oxygen atoms lead to form partial negative charges. This indicate that glucose should have partial charges but they are not like salts with full charges. A conclusion of above results and explanations about cell's membrane; Polarity factor is more important for

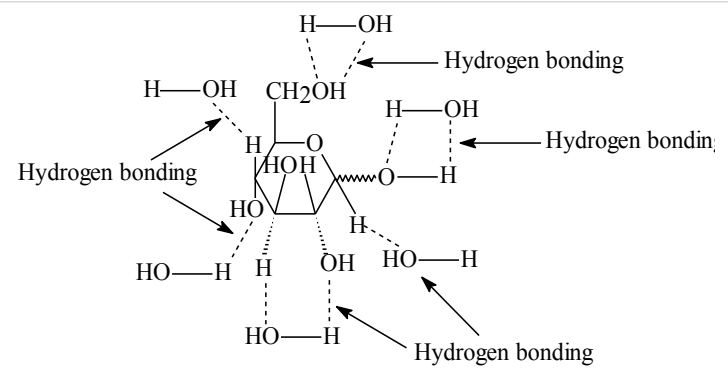

Figure 7: Showed hydrogen bonding between glucose and water molecules. molecules to penetrate through them but there are another factors should control penetration process such as hydrogen bonding and solubility of different molecules such as glucose in the blood.

According to chemistry rules sodium chloride molecules are not dissolve in oil due to they are polar molecules and oil is not polar "like dissolve like" but even that they can pass through oil and keep moving within so easily. This behavior is unbelievable for these polar molecules but must be there strong forces forcing them for passing through oil and keep moving. These forces can be illustrated as different salts represent top polar compounds this why preparing most drugs as salts rather than their original organic structure because salts can penetrate through different oil and hydrolyze in aquatic solvents. Repulsion between salts and oils let salts move in different oils, which means, this behavior make salts able to do a path through different oils due to repulsion that forced molecules of different oils to be a way from salts let them pass through. Therefore, glucose as sodium Gluconate is much better than glucose in penetration process through membranes of different cells.

Sodium chloride and glucose pass through different layers but other molecules cannot! This actions are not belong for different layers, it belongs to different molecules that passing through them. In another words, chemical properties of different molecules are responsible for making them passing through oil's layers so this action is not for these layers it is for molecules themselves. Therefore, bilayers of "cell's membrane" do not have the choice to select which ions or molecules should pass through, this choice is for chemical properties of different ions and molecules that passing through. These membranes have certain molecules and passing molecules have different molecules so passing processes are depend on chemical properties of both molecules.

Another point is that human cells are not place each one alone, they arranged each one connect to the others to form different tissues in human's body. Therefore, may be $0.25 \%$ of all cell diameter that contacting with blood while the rest $0.75 \%$ is contact with neighboring cells. In addition, there are another cells in different human's tissues do not contact with blood. This means that each cell that contact with blood has very short distance (about $0.25 \%$ ) to exchange important ions and molecules with blood and then giving these ions and molecules to neighboring cells, this cell like a bridge for transferring important ions and molecules from blood to neighboring cells that are not contact with the blood. Then through this short distance that is contact with blood, there are high amounts of many ions and molecules should pass.

It should be noticed that after passing of negative compounds through cell's membrane, membrane's molecules return to them original structure, cell's membrane return 
to its shape or relatively return to its shape because they are moving continually. However, this for lipid part and hydrophobic part of cell's membrane because protein part and hydrophilic part may have either positive or negative charges so penetration activity should be depended on them charges and on anther factors.

Each situation have its factors and this research is about how glucose enter human's cells so situation of glucose factors is explained very well in above explanations. Sodium Gluconate is better than glucose in penetration through cell's membrane because Gluconate ion have negative ion easy to penetrate through cell's membranes as explained for sodium chloride salt.

This is what this research searching, other ions or molecules that entering human cells should have same or different explanation depending on all factors may affect penetration process.

Action of potassium ions in entrance of glucose inside human's different cells: This the last step of how glucose enter human's cells, insulin convert glucose to be as a salt "sodium Gluconate", then these conversion results enter inside different human cells. This process depend on sodium ions as it is mentioned before. Therefore, Potassium ions have a major important action in this operation because they enter the cell and due to this entrance three sodium will exit [1-4]. Therefore, potassium ions action is to prepare sodium ions for producing Gluconate ion. Sodium ions have two importance actions; activate insulin to react with glucose and make Gluconate ions more stable for entering different cells.

Insulin concentration in blood have to be enough for glucose concentration so insulin molecules should catch glucose molecules and reacting with. Glucose should remove hydrogen bonds that occurred between its molecules with different molecules of the blood, and it should leave blood as a salt more soluble. Glucose has high solubility means that it prefers to stay in blood rather than leave it.

Gluconate ions entering human cells to react with Adenosine Triphosphate (ATP). The main reaction that occur can be illustrated as in the following scheme (3):

Scheme (3): Mechanism of Gluconate reacting with ATP molecules.

By collecting all three points of how glucose enter human's cells and their facts, the brief of glucose process can be explain as:

The first step is most important for all glucose entrance inside human's cells because potassium ions concentration inside human cells are thirty times than its concentration outside human cells [1-3]. Therefore, human must eat food, vegetables and others that are rich in sodium and potassium ions for providing its blood by these ions. Between human meals, potassium ion concentration decrease because absence of its sources. Equilibrium forces work in both sides, before the meal potassium level inside the cell is higher than its level in blood. Therefore, different cells lose some of its potassium to reach equilibrium point and this happen due to equilibrium forces. In contrast, after each meal potassium concentration outside the cell should be higher than inside so these ions penetrate through cell membrane to reach equilibrium point.

References indicated [1-4] that there are certain amounts of sodium ions in the blood outside human cells but these amounts are not enough for glucose-insulin reaction because each insulin molecule need (13) sodium ions so sodium ions that eliminating from human cells are so important for glucose to enter inside body cells rather than outside ions.

Finally, concentration of producing (13) sodium Gluconate should be higher than its concentration inside human cells so equilibrium forces must push these products inside human different cells through cells membranes. Sodium Gluconate is active molecules so they should react with Adenosine triphosphate (ATP) to produce first compounds of glycolysis. Glucose-6-phosphate and Adenosine Diphosphate (ADP), as illustrated in above scheme and as it is indicated in the following equation:

$\left(\mathrm{C}_{6} \mathrm{H}_{11} \mathrm{O}_{6}\right)^{-} \mathrm{Na}^{+}+\longrightarrow$ ATP Glucose-6-phosphate + ADP .... (7)

Glucose-6-phosphate convert to be fructose-6-phosphate and keep converting in glycolysis until converted to; necessary energy for human's body, $\mathrm{CO}_{2}$ and water molecules.

There is another additional explanation, scientists indicate that potassium ions have major importance inside human cells while sodium ions have major importance outside human cells [1-8,10-14]. Furthermore, as it is mentioned before, references [1-4], showed that there is active ions exchange happened between inward and outward human's cells called sodium-potassium $\left(\mathrm{Na}^{+}-\mathrm{K}^{+}\right)$pump.

When potassium ions in human's blood are not enough then main reaction should occur and concentrations of glucose, ammonia and hydrogen ions should increase. In addition, when potassium ions are not enough, equilibrium forces work in contrast of above action then human cells eliminate potassium ions for reaching equilibrium point.

This means that human's cells should release potassium ions instead of take them so concentration of potassium ions have increase in human body in addition to above ions and molecules (glucose, ammonia and hydrogen ions). As mentioned before, references indicated that diabetes patients have high concentration of glucose, ammonia, acidity and potassium ion [1-4,7-13].

Human's body need large amounts of potassium ions 
in its foods because after they taking these ions, glycolysis processes inside human cells happen, after that the excess of these ions must eliminate by kidneys. About $92 \%$ of eating potassium ions are removing by kidneys [3].

This process of entering glucose inside human's cells is so clean and perfect, $100 \%$ of eating potassium is just for helping potassium ions to enter human's cells for producing 3 sodium ions for binding with 3 Gluconate ions. This because concentration of potassium ions inside human's cells is thirty times than its concentration outside cells therefore $100 \%$ of potassium ions are just for helping Gluconate to enter human cells due to equilibrium forces. This means that human cells take only $8 \%$ of $100 \%$ from eating potassium ions while the rest $92 \%$ should be removed by kidneys. This elimination ratio of potassium should circulate in water sources from one person to another but when they drink commercial waters! This does not happen because different filters eliminate potassium ions due to their big volumes. This the reason why diabetes has happen in high ratio for the last decades due to most people use commercial waters which are empty from potassium ions.

A conclusion, Potassium ions are so important in glucose entering inside human's cells so any decreasing in them must decrease producing of sodium Gluconate leading to increase glucose in the blood in addition to increase; ammonia, $\mathrm{H}^{+}$ ions, and potassium ions. This means that decreasing of potassium ions that enter human's body with its foods and others should cause diabetes disease.

Before explaining additional points more than what it is mentioned before, there is more important point should be noticed about how glucose spread through body's different tissues.

As it is mentioned before, human's cells are connect each other and not all of them are adjacent to blood paths so equilibrium forces make each cell have same molecules or atoms as others of human's body by pushing these important elements through cells membranes until all cells get same amounts. After each meal equilibrium forces work until each cell whatever its position in the body should take same ions and compounds that needs for continue in its living systems.

In diabetes disease, potassium ions are not enough for all body's cells, there are a number of these cells do not get these ions means sodium ions stay inside different cells so above processes have not take place. Therefore, glucose molecules stay in the blood as they are leading to increase them concentration, concentration of ammonia molecules should increase too from hydrolysis of insulin leading to increase urea concentration in the blood, finally acidity of blood that come from insulin hydrolysis should decrease unless giving the patient special treatment [1-3]. Moreover, as it is mentioned before, potassium ion increase in patient's blood for getting equilibrium point between inside and outside patient's cells.
This should effect blood pressure because this pressure depend on sodium ions at 90\% [1-3] and there is a relation between sodium and potassium in $\mathrm{Na}^{+}-\mathrm{K}^{+}$pump [1-3]. Then sodium/potassium concentrations are so important for blood pressure and for diabetes therefore any disorder in concentrations of above ions in human's cells must lead to change blood's pressure or cause diabetes. This change should depend on concentration of potassium ions that enter human's body for entering body's cells leading to eliminate sodium ions which are responsible for blood pressure. In another words, low concentration of potassium ions in the foods lead to decrease sodium ions and increase potassium ions leading to higher blood pressure because potassium is bigger in atomic size than sodium causing high pressure on veins and arteries leading to hypertension and also increase of potassium ions will effect human heart leading to hypertension too.

In fact, any disorder in human's body should affect other systems of this body. Such as; potassium ion has special importance in many body's systems, one of them is; its relation with aldosterone of sexual system so any disorder in potassium ions will effect sexual system. Therefore, changing in potassium system in glucose entering inside human's cells lead to many changes in human body. Equilibrium forces organized human body in perfect picture so any changes in this equilibrium must lead to change body's equilibrium leading to many diseases such as diabetes, high blood pressure and others.

For confirming this research results and explanation about importance of potassium ions for human life there are three good studies indicate that the low levels of serum potassium increase Diabetes risk [14-17]; First study, 12209 persons were involved for about 27 years 1985-2012 [15]. Second study is a comparison between African Americans and white Americans, it showed that Africans are more than whites in type (2) diabetes [16]. The last study was involved 4409 Japanese men for about (4) years and its results are same as above studies that low levels of serum potassium increase risk of diabetes [17]. This means that above studies took more than 16618 participated persons for long time more than 30 years 1985-2014 for getting a result that low serum potassium increase diabetes risk.

These studies got those results according to practically methods and their results are as same as this research's results. Unfortunately, above studies did not find the right explanation for them results or they do not find the importance of serum potassium in diabetes type 2 disease, while this research find the right explanation for all results its results and above studies results as well, this was shown in this research for the relation between low serum potassium ion with increasing risk of Diabetes type 2 disease. As it is mentioned before, Potassium ions are thirty times inside different cells than outside, then these ions should be inside different cells rather than getting out. 
One of Diabetes signs is high amounts of urine, and this because that when potassium ions are not enough, human's cells release potassium ions instead of taking them, and this releasing should occur to water molecules with potassium ions. In addition, sodium ions stay in the blood without any changing. Therefore, sodium and potassium ions be outward patient's cells, higher in concentrations than inward patient's cells so osmotic pressure must force water molecules to be out patient's cells which means these molecules be in the blood. Increasing of water molecules in the blood make kidneys eliminate them by increasing urine. This is not the only effect, diabetes patients have less sizes than normal people and this because that them cells are continually losing water molecules by osmotic pressure as indicated above so they should be less water molecules leading to less sizes. There are another effects of decreasing of potassium ions but they are out of this research scope.

Over the world there are many herbal treatment and before about two months a Arabic expert showed that mixing onion with honey is the best treatment for diabetes. This is experimental treatment which means many people took it before and they found it useful treatment. The fact of this is that this treatment according to this study is the best treatment because onion and honey are very rich in potassium ions. In fact, all fruits, vegetables, herbs and others that used as diabetes treatments are good treatment because all of them contain a certain high amounts of potassium ions so they are good treatments for diabetes. It should be noticed that potassium concentrations in above fruits, vegetables, herbs and others are differ from one kind to another.

In fact, above fruits, vegetables, herbs and others are normal treatment for diabetes because they may have another ions or compounds that treating diabetes except honey which is more argumentative treatment because it is high amount of sugar and diabetes means a high sugar in the blood! It is like dropping oil on fire but even that experimental results indicated that honey is a good treatment for diabetes. According to the science, there is not an explanation for honey to be a good treatment for diabetes! While the best explanation for this, this study which indicated that honey contain high amounts of potassium ions therefore it is a good treatment for diabetes.

Different foods are not only sources for potassium ions, tap water or drinking water is a good source for these ions (according to results of this research tap water contain about $3.1-3.2 \mathrm{mg} / \mathrm{L}$ of potassium ions), and also human take drink water (tap water) many times more than three times in the day rather than three times of taking different meals. This means that drinking water provide human body by potassium ions continually many times in the day. Unfortunately, nowadays many people around the world drinking filtered water with no potassium ions!
Ionic radii of Potassium ion $\left(\mathrm{K}^{+}\right)$is $1.33{ }^{\circ} \mathrm{A}$, it is bigger ion so it cannot pass through simple filters. This research results indicate this fact and it is; Potassium ions cannot pass through different filters even simple one because of its size. Therefore, filtered water does not contain potassium ions or it contains not enough.

In addition, by cooking different foods all of them losing potassium ions which means cooking foods containing less potassium ions rather than not cooking foods. Therefore, filtered water and cooking foods should cause diabetes, high blood pressure and relating diseases because they have less potassium specially if the person does not eat foods with high potassium levels such as different fruits or different vegetables or others. Diabetes is not a disease, it is unordered and unbalancing in different ions of foods or others that taken daily.

There is no relation between fat people with diabetes type 2 or with hypertension diseases, it just that these people have more cells than other people then they need more ions (potassium or sodium) than other normal people. It is a fact, that if normal people do not get enough ions due to their drinking water or their different foods so what about fat people?

Therefore, fat people for avoiding diabetes type 2 and different blood's pressures especially hypertension, they must get more ions potassium and sodium than other people in them different foods or drinks. As example if it is enough for normal people getting one apple then fat people must get more than one apple such as two or one and half apple. This the fact of fat people with above diseases and with other diseases they have more cells need more than other people to be healthy person.

In Missan people drink filtered water they called it "Reverse Osmotic water", this water does not contain potassium ions, in addition Missan's people cook them foods and them tea, milk and other solutions by it. Because this there is a new disorder or disease was happened in this city which is, new born babies are come to this life with diabetes disease, this unusual disease but it is happened in Missan and other relating cities. This because that mothers of these babies do not get enough potassium for them bodies so how they give potassium to them babies!? These cases happened for women do not get enough potassium ions in them foods.

According to this research, diabetes does not have two or three types, it is a result of decreasing in potassium that taken during human life in every day. Therefore, there are two cases; firstly if both concentrations of glucose and insulin is normal in human's blood then the reason of diabetes is low levels of potassium ions which enter human's body. The second case, if insulin concentration is less than normal or there is no insulin in human's blood then this means that the reason of diabetes is $\beta$-cells. 
Therefore, insulin concentration in human's blood should be the right tested instead of glucose tests because glucose concentration is always normal or up to normal in the blood. This indicates that people must check insulin concentration in them bodies by different laboratories rather than measuring glucose concentration.

Measuring of insulin concentration in human's blood give two results either high or normal levels, then this means diabetes come from low levels of potassium ions. Or low insulin levels in the blood then $\beta$-cells are at abnormal status something wrong with them.

This research mentioned many facts and good evidences for showing the right treatment for diabetes and hypertension diseases such as; A high ratio from new born babies that come with diabetes (this research samples), people of AlMejar Al-Kabeer that are about 70000 having a high diabetic ratio, chemical facts of insulin, glucose and cell's membrane, chemical explanations of other aspects, ...etc. Following facts are differ than these facts of this research because they are unbelievable and it is so difficult to understand why they do this!

Doctors and specialists [19], have indicated that at emergency statuses of diabetes, hypertension and hypotension they have specific protocols should be followed; For diabetic patients may be over $400-500 \mathrm{mg} / \mathrm{dl}$ of serum glucose for both; old and young patients: Firstly they give them injection insulin and wait for a while, if the glucose levels does not fall down to normal statuses then they give them normal saline which is sodium chloride solution $(\mathrm{NaCl})$. This must decrease glucose level for being at normal case but they mentioned additional points that they must control concentration of this salt with the size of the patient because for many cases above method decrease glucose level to dangerous statuses they give the patients solution of glucose with potassium, this should increase glucose level to normal levels. In addition, These specialists indicates that there are another cases caused death for many cases because normal saline cause stroke leading to death mostly for both old or young patients.

These information are unbelievable and surprising because they are exactly same as this research results so those specialists were asked many times from where they got these information! Are they got these information from previous study of Al-Darraji study at 2013 which have same results of this research results but they were told that they do these methods before long time ago. After insisting they were told that they got these ways from medicine's books and the doctors and specialists follow these books!!

As it is this ugly crime how these books contain the right treatment for diabetes before long time but no one know about them or the doctors and specialists do not tell patients people, everyone and all the world about this treatment!
Absolutely they know what they do and their books have the right treatment for more dangerous disease that millions of people suffering from, this behavior all the words cannot explain.

However, it is important to mentioned this before explain above information; Insulin is forming from just amino acids (proteins) so human's body need a huge amounts from these acids each day. It is true that insulin does not change after reacting with 11 glucose molecules but it is half life time is too short so there is always some of insulin molecules Sometimes specially old people that they do not eat protein in sufficient amounts therefore them bodies cannot release enough insulin causing diabetes type 2 and them treatment is injection insulin for recovering the insufficient amounts.

Above medical information were so clear that bloods of these patients have insulin, glucose and potassium but they do not have sodium ions so the glucose stay in these bloods to be much higher than normal statuses. Normal saline or sodium chloride is the right treatment for this conditions because potassium ions cannot bind to glucose while sodium ions can. Therefore, with assistance of insulin Glucose-sodium molecules should formed to enter different cells rather than stay in the blood as it is mentioned before. This right treatment was known for long time ago and it is a protocol the doctors and specialist follow in different hospitals for diabetes patients.

Whereas, for many patients sodium chloride reduce glucose concentration but it causes hypertension leading to death. This reason is so known before so long time ago that this salt increase blood pressure because when sodium ions enter different cells for each three ions about 2 potassium ions get out. Potassium ions are bigger than sodium so they cause pressure on different walls of arteries and veins leading to hypertension.

Finally, filtered water (such as R.O. water), Cooking foods, continue eating foods with low potassium ions and others factors should cause diabetes, high blood pressure and relating diseases. Above factors are just faces of our civilization, therefore, civilization is the main reason of diabetes, high blood pressure and relating diseases.

For confirming this research results and explanation about importance of potassium ions for human life there are three good studies indicate that the low levels of serum potassium increase Diabetes risk [14-17]. First study, 12209 persons were involved for about 27 years 1985-2012 [15]. Second study is a comparison between African Americans and white Americans, it showed that Africans are more than whites in type (2) diabetes [19]. The last study was involved 4409 Japanese men for about (4) years and its results are same as above studies that low levels of serum potassium increase risk of diabetes [17]. This means that above studies took more than 16618 participated persons for long time 
more than 30 years $1985-2014$ for getting a result that low serum potassium increase diabetes risk. Unfortunately, these studies do not explain their results but this study did this in above good and strong evidences and explanations that balancing of important ions sodium and potassium with enough proteins each day are important for getting good health away from diabetes, blood pressure and other diseases.

\section{Conclusion}

Diabetes, hypertension and similitude or relating diseases are not ordinary disorders therefore this research need to discover the right treatments for them by more than four different scientific ways; Firstly, according to 70000 samples of a small city for the last 20 years nothing change in them lives except they change them drinking water for this diabetes type two ratio increased from about 2-3\% before 2003 to $28.5 \%$ after 2003 . Secondly, science reach that sodium ions enter with glucose molecules through cell's membrane but it does not show the bond type between these two chemicals is it ionic or covalent bond! Thirdly; according to chemistry rules this research explain what above bond is, resulting find the right treatment for most unlikely diseases. Fourthly; after finishing this research three manuscripts [1517], were found that have same of this research points that low serum potassium cause diabetes type two. In addition, there are another additional evidences lead this research to discover the right treatment for; diabetes, hypertension and relating diseases.

\section{References}

1. Saladin KS, Porth CM. Anatomy and Physiology, The unity of form and function. McGraw-Hill companies. 1998.

2. Seeley RR, Stephens TD, Tate P. Anatomy and Physiology. Seventh Edition, McGraw-Hill companies. 2006.

3. Arthur Guyton C, John E. Hall Text book of Medical Physiology. Eleventh Edition, Elsevier Inc. 2006.

4. Garrett RH, Grisham CM. Biochemistry Fourth Edition. 2010.

5. Sodium - A comprehensive Analysis. 2012. http://www.abcbodybuilding.com/magazine03/sodium.htm

6. Ellert M. Nutrient Absorption. 1998. http://www.siumed.edu/mrc/research/nutrient/gi42sg.html.
7. Yahoo voices. Five causes of a high potassium level. 2007. http://voices.yahoo.com/five-causes-high-potassium-level-485267. html?cat=5.

8. Colledge NR, Walker BR, Ralston. Davidson's Principles and of Medicine. 2010.

9. Bookallil MJ. University of Sydney Nuffield-Department of Anaesthetics lectures and study notes, Causes, Diagnosis and Effects of abnormal pH status. 2011.

http://www.anaesthesia.med.usyd.edu.au/resources/lectures/ acidbase_mjb/causes.html

10. Lal SS. Hyperuricemia, High serum Urea and hypoproteinemia are the risk factor for Diabetes. Asian J Medical Sci. 2009; 1: 33-34.

11. Deepa K, Manjuntha Goud BK. Serum Urea, Creatinine in relation to fasting plasma glucose levels in type 2 Diabetic patients. Int J Pharm and Biolog Sci. 2011; 1: 279-283.

12. Blessing $\mathrm{O}$, Idonije OF, Olarewaju MO. Plasma glucose, creatinine and Urea levels in type 2 Diabetic patients attending a Nigerian Teaching Hospital. Res J Med Sci. 2011; 5: 1-3.

13. Ranee Ch, Hsin-Chieh $Y$, David E, Frederick B. Potassium and risk of type 2 diabetes. Expert Rev. 2011; 6: 655-672.

14. Richards BJ. Low levels of Potassium linked to increase Diabetes risk. Wellness Resources. 2011.

http://www.wellnessresources.com/health/articles/low_levels_of potassium_linked_to_increased_diabetes_risk/

15. Chatterjee R, Yeh HC, Shafi T, Selvin E, Anderson C, et al. Serum and dietary potassium and risk of incident type 2 diabetes mellitus: The Atherosclerosis Risk in Communities (ARIC) study. Arch Intern Med. 2010; 170: 1745-1751.

PubMed: https://www.ncbi.nlm.nih.gov/pubmed/20975023

16. Chatterjee R, Yeh HC. Potassium and the Risk for Type 2 Diabetes African-American Study. J Clini Nutrition. 2011.

17. Heianza $Y$, Hara S, Arase $Y$, Saito $K$, Totsuka $K$, et al. Low serum potassium levels and risk of type 2 diabetes: the Toranomon Hospital Health Management Center Study 1. Diabetologia. 2011; 54: 762-766. PubMed: https://www.ncbi.nlm.nih.gov/pubmed/21212932

18. Murray RK, Bender DA. HARPER'S Illustrated Biochemistry. $29^{\text {th }}$ edition. The McGraw-Hill. 2012.

19. Personal communications with doctors and specialists at different hospitals of Missan city. 2018-2019.

20. Al-Darraji AH. Diabetes, blood pressure, and relating diseases; reasons and solutions. Eur J Scientific Res. 2013; 104: 1. 